\title{
SINGLE EVENT EFFECTS IN ANALOG-TO-DIGITAL CONVERTERS: DEVICE PERFORMANCE AND SYSTEM IMPACT
}

\author{
T. L. Turflinger, M. V. Davey, B. M. Mappes ${ }^{2}$ \\ Crane Division, Naval Surface Warfare Center \\ Crane, Indiana 47522
}

\begin{abstract}
Monolithic Analog-to-Digital Converters (ADCs) exhibit a large error rate in the single event effects (SEE) environment. Analysis of data from a high-performance ADC demonstrates the type of errors and their potential impact on system performance.
\end{abstract}

\section{INTRODUCTION}

While SEEs in memory devices have been well characterized, the system impact of SEE in other complex electronic devices is not effectively characterized. This effort demonstrates that high-performance ADCs, which are used extensively in modern satellite systems, do indeed have a substantial SEE-induced error rate and that the error rate can be effectively characterized using reasonably simple modifications to well-known error rate calculations as used for memory devices. The orbital error rate for a radiationhardened, 12-bit, 5-Million Sample Per Second (MSPS) ADC, the Analog Devices AD -42961, has been experimentally determined and is used to demonstrate the potential impact of ADCs on system error rates. Limited proton data is also discussed. In addition, the paper demonstrates that the error rates calculated using previously established test methods and data analysis techniques $[1,2]$ are independent of variations in device parameters (supply voltages, input voltage) and the flux or ion rate of the ionized particle beam. It is further shown that portions of the SEEinduced errors in the ADC are caused by the analog portions of the ADC and, thus, provide insight into the effects of analog SEE. The potential impact on system performance is severe if consideration of this error source is not given during system development.

\section{ANALOG DEVICES AD-42961}

The AD-42961 is a commercially available, radiationhardened, 12-bit, 5-MSPS ADC that consumes 1.2 watts of power, using $+/-5$ volt supplies and $a+/-1$ volt input voltage range. The device is fabricated in Analog Devices radiation-hardened BiCMOS technology, RBCMOS, which was developed in conjunction with PMA-A1104 of the US Army Space and Strategic Defense Command. The
$\mathrm{AD}-42961$ is a radiation-hardened variation of the nonhardened commercial AD-871 [3]. A block diagram of the AD-42961 is shown in Figure 1. The architecture of the device is a four-stage, feed-forward, series-parallel converter with integral track-and-hold amplifiers. The integral voltage reference is a carry-over from the commercial AD-871 and utilizes a band-gap design that does not maintain superior radiation performance. For use in a radiation environment, an independent radiation-hardened reference should be used. Electrically, the AD-42961 demonstrates excellent dynamic performance with a pre-radiation signal-to-noise ratio (SNR) in excess of $68 \mathrm{~dB}$ (11.2 effective number of bits (ENOB), Differential Non-Linearity (DNL) of $1.0 \mathrm{lsb}$ (least significant bits), and Integral Non-Linearity (INL) of $1.5 \mathrm{lsb}$ ). Preliminary total dose tests on the AD-42961 show no performance degradation below $500 \mathrm{kRad}(\mathrm{Si})$. Neutron degradation has been shown not to be a problem for the RBCMOS technology. The device does not latch in any tested environment, including Xenon ions at a beam angle of $60^{\circ}\left(\mathrm{LET}=126.6 \mathrm{MeV}-\mathrm{cm}^{2} / \mathrm{mg}\right)$ with a fluence of $1 \mathrm{E} 7$ ions $/ \mathrm{cm}^{2}, 165 \mathrm{MeV}$ Protons at a fluence of $1.2 \mathrm{E} 11$ ions $/ \mathrm{cm}^{2}$, and electron beam dose rate greater than $1 \mathrm{E} 11 \mathrm{rads}(\mathrm{Si}) / \mathrm{sec}$. The AD-42961 is the highest known performance radiationhardened ADC available in the world.

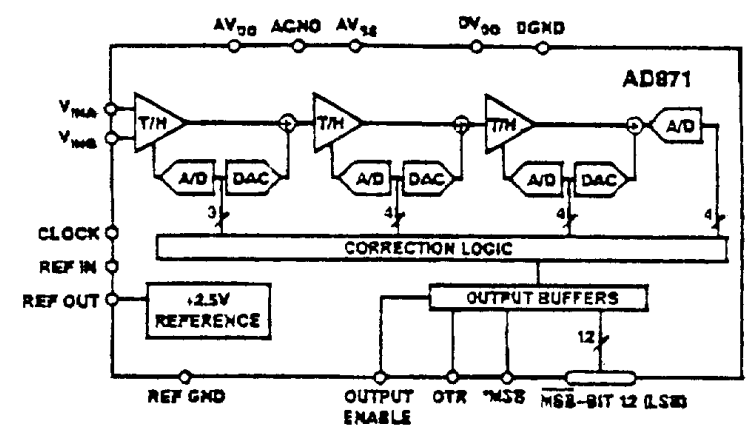

Figure 1. AD-42961 Block Diagram

\section{TEST OVERVIEW}

Experimental data were collected at the 88-inch Cyclotron at Lawrence Berkeley Laboratory, using the Aerospace Chamber. Data were collected with six ions at normal incidence, as listed in Table 1 below.

\footnotetext{
${ }^{1}$ Work sponsored by USASSDC.

${ }^{2}$ Now with Burr-Brown, Inc.
} 


\begin{tabular}{|c|c|c|c||}
\hline \multicolumn{4}{|c|}{ TABLE 1: ION LIST } \\
\hline Ion & $\begin{array}{c}\text { LET } \\
M^{*} \cdot \mathrm{cm}^{2} / \mathrm{mg}\end{array}$ & $\begin{array}{c}\text { Energy } \\
\mathrm{MeV}\end{array}$ & $\begin{array}{c}\text { Range } \\
\mu \mathrm{m}\end{array}$ \\
\hline $\mathrm{N}$ & 3.2 & 67 & 121 \\
\hline $\mathrm{Ne}$ & 5.6 & 90 & 52 \\
\hline $\mathrm{Ar}$ & 15.0 & 175 & 43 \\
\hline $\mathrm{Cu}$ & 30.0 & 283 & 43 \\
\hline $\mathrm{Kr}$ & 40.0 & 366 & 47 \\
\hline $\mathrm{Xe}$ & 63.3 & 603 & 52 \\
\hline
\end{tabular}

During the test, a total of three devices were exposed. Table 2 lists the variations in device bias, input voltage, clock rate and ion flux used to verify device cross-section independence from test parameters. A data set is defined when data on at least five of the six ions are collected at the same electrical conditions. Single event latchup screens, with Xenon at a beam angle of $60^{\circ}$ and maximum power supply voltages, displayed no latchup with a fluence of $1 \mathrm{E} 7 \mathrm{ions} / \mathrm{cm}^{2}$.

\begin{tabular}{||c|c|c|c|l||}
\hline \multicolumn{5}{|c|}{ TABLE 2*: Data Set Parameters } \\
\hline SN & $\begin{array}{l}\text { Bias } \\
\pm V\end{array}$ & $\begin{array}{l}\text { Vin } \\
\text { V }\end{array}$ & $\begin{array}{l}\text { Clock } \\
\text { MSPS }\end{array}$ & $\begin{array}{l}\text { Flux } \\
\text { \#/sec }\end{array}$ \\
\hline 1 & 5 & 0.0 & 5 & $3.125 \mathrm{E} 4$ \\
\hline 2 & 5 & 0.0 & 5 & $3.125 \mathrm{E} 4$ \\
\hline 2 & 5 & -0.75 & 5 & $3.125 \mathrm{E} 4$ \\
\hline 2 & 5 & +0.75 & 5 & $3.125 \mathrm{E} 4$ \\
\hline 2 & 5 & 0.0 & 5 & $3.125 \mathrm{E} 3$ \\
\hline 2 & 5 & 0.0 & 5 & $1.573 \mathrm{E} 5$ \\
\hline 3 & 5 & 0.0 & 5 & $3.125 \mathrm{E} 4$ \\
\hline 3 & 5 & 0.0 & 1 & $3.125 \mathrm{E} 4$ \\
\hline 3 & 5 & 0.0 & 2.5 & $3.125 \mathrm{E} 4$ \\
\hline 3 & 4.75 & 0.0 & 5 & $3.125 \mathrm{E} 4$ \\
\hline \hline
\end{tabular}

* Data in Table 3 is in same order.

The test methodology utilized to collect unambiguous data has been previously described by the authors [1]. Unique PC-based test hardware [4] was utilized, which allowed continuous collection of dynamic ADC data in excess of one billion samples. For these experiments, each data point represents 300 million (3E8) samples of data dynamically collected while the part was in the particle beam. In this manner, statistical uniformity was achieved, as will be demonstrated. Beyond the continuous data collection allowed by the test hardware, other key features of the test method include utilizing a DC input voltage to allow unambiguous separation of SEE-induced errors from expected data, dynamic exercise of the device while in the particle beam, and use of histograms or probability density functions (PDFs) to collect and display the test data.

\section{DATA ANALYSIS}

Data collected from the test ADC are plotted in a histogram, or probability density function (PDF), where the 4096 possible digital output codes are on the $\mathrm{x}$-axis and the number of occurrences of each code is on the y-axis [1]. Immediately preceding the irradiation, a pre-radiation measurement provides the expected data, which is subtracted from the irradiation data, leaving only the SEE-induced errors [2]. In the test fixture used, the pre-irradiation data of the AD-42961 typically had data in five output codes (or bins) for a given DC input, with most expected data occurring in the center three bins. An ideal converter would have data in only one bin, given an ideal DC input. Figures 2 through 7 show sample experimental data PDFs for the AD-42961 with an input voltage of 0.0 volts (mid-scale) and a conversion rate of 5 MSPS. Data from each of the six ions listed in Table 1 are shown sequentially in the figures.

Several comments should be made about the qualities apparent in the SEE-induced errors shown in the figures. First, the vertical axis of the graphs have been "clipped" at 200 errors so that small error counts can be observed. A few bins near the expected data reach the maximum count of 4095 errors. Second, the expected data were contained in five bins near the center (bin 2048) of the displayed scale, and have been subtracted from the SEE-induced errors. Physical limitations of the graphs prevent this open space occupied by the expected data from being observed, but only SEE-induced errors are displayed in these figures. The data with \pm 0.75 -volt inputs have similar characteristics, but the expected data are offset at the appropriate output code. Third, there is a distinct cluster of SEE-induced errors around the expected data. It has been shown [2] that the distribution of these errors is Gaussian in nature. In addition, other errors can be seen which clearly do not fit the Gaussian. Even with Nitrogen ions (Figure 2, LET $=3.2$ $\mathrm{MeV}-\mathrm{cm}^{2} / \mathrm{mg}$ ), a small but significant, number of errors fall well outside three standard deviations; one recorded error is at the maximum possible output code of 4095 (where the expected data was mid-scale, or 2048). The occurrence of these "non-Gaussian" errors is apparent in the other graphs. The tendency of the non-Gaussian errors to group at certain codes indicate saturation of one or more of the four internal 


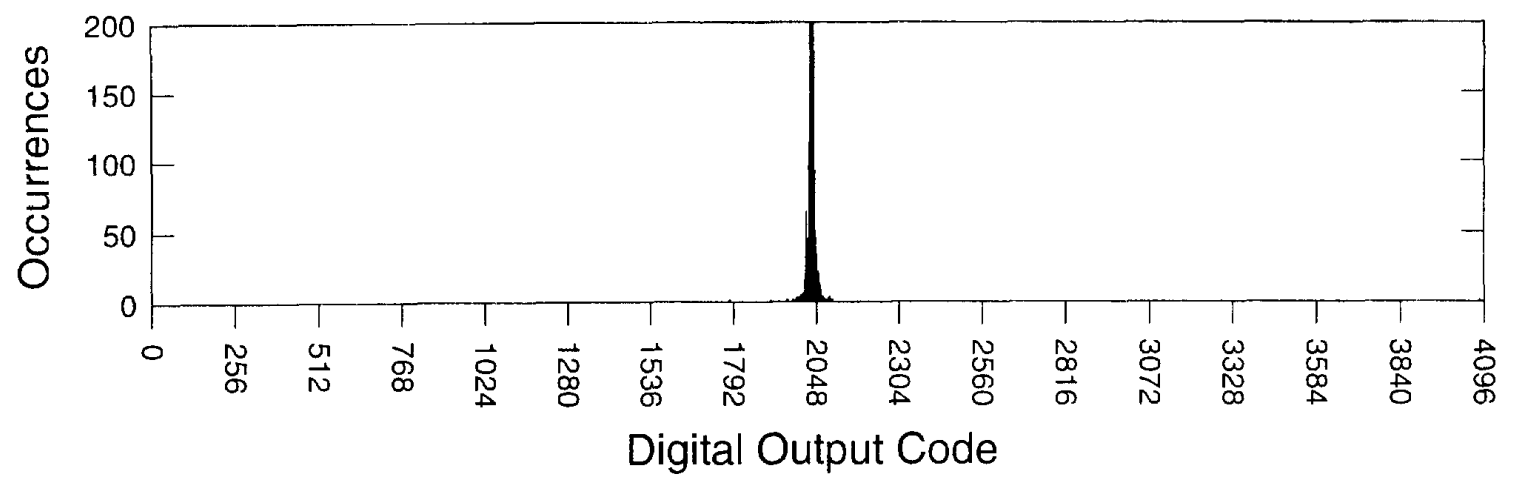

Figure 2. AD-42961 SN3302: $\mathrm{V}_{\mathrm{IN}}=0.0 \mathrm{~V}, \mathrm{~F}_{\mathrm{Cl.K}}=5 \mathrm{MSPS}$

Nitrogen Ions, $\mathrm{LET}=3.2 \mathrm{MeV}-\mathrm{cm}^{2} / \mathrm{mg}$

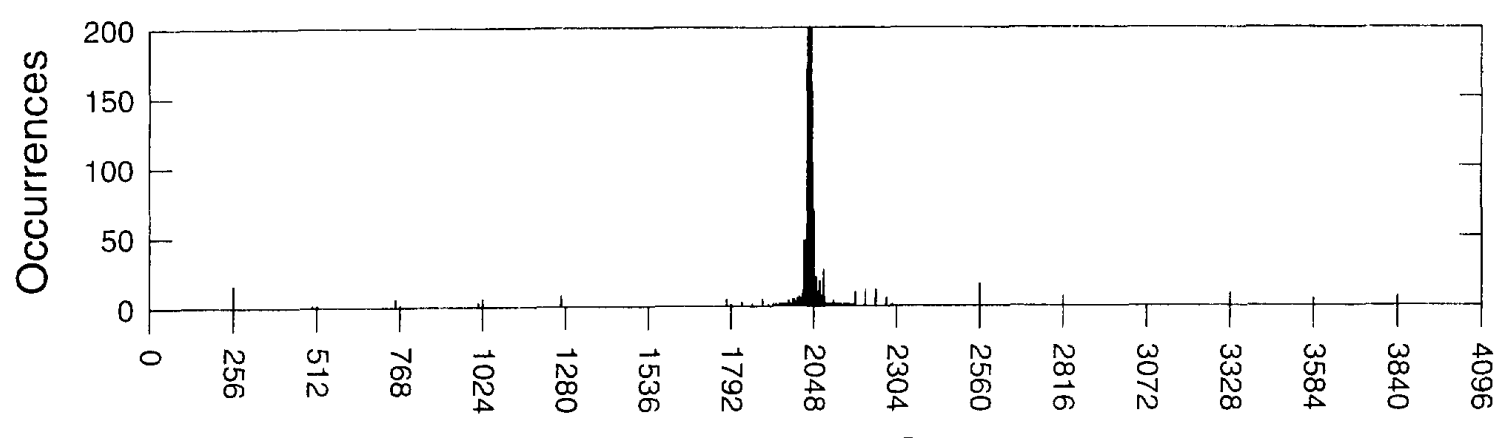

Digital Output Code

Figure 3. AD-42961 SN3302: $V_{I N}=0.0 \mathrm{~V}, F_{C L K}=5 \mathrm{MSPS}$

Neon Ions, $\mathrm{LET}=5.6 \mathrm{MeV}-\mathrm{cm}^{2} / \mathrm{mg}$

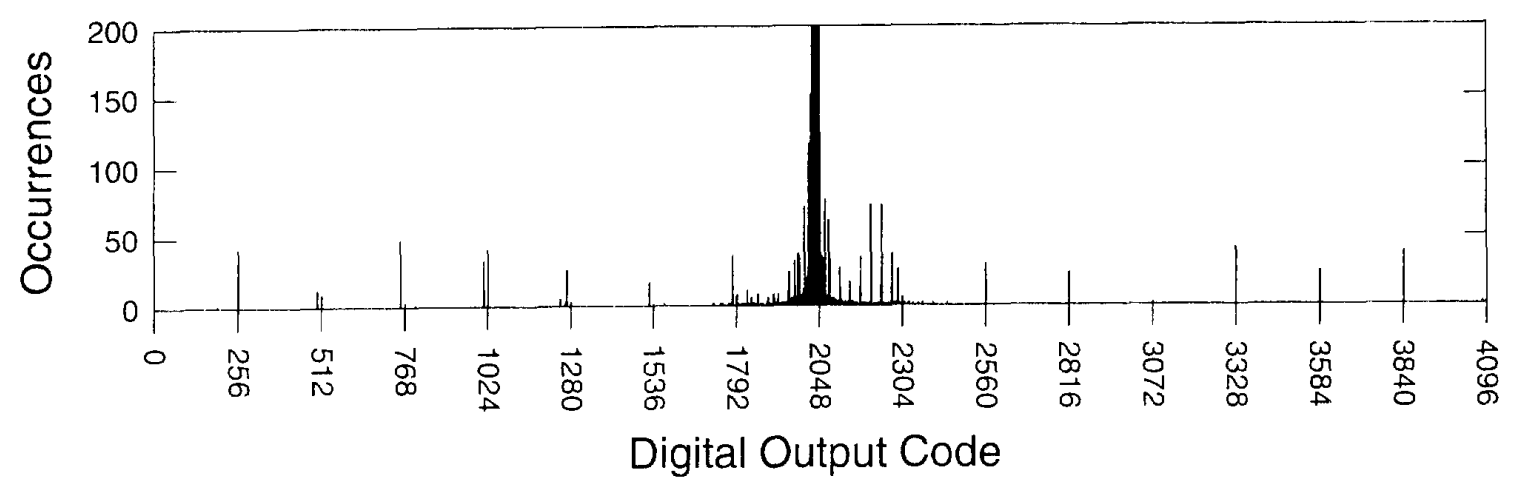

Figure 4. AD-42961 SN3302: $\mathrm{V}_{\mathrm{IN}}=0.0 \mathrm{~V}, \mathrm{~F}_{\mathrm{CLK}}=5 \mathrm{MSPS}$

Argon Ions, $\mathrm{LET}=15 \mathrm{MeV}-\mathrm{cm}^{2} / \mathrm{mg}$ 


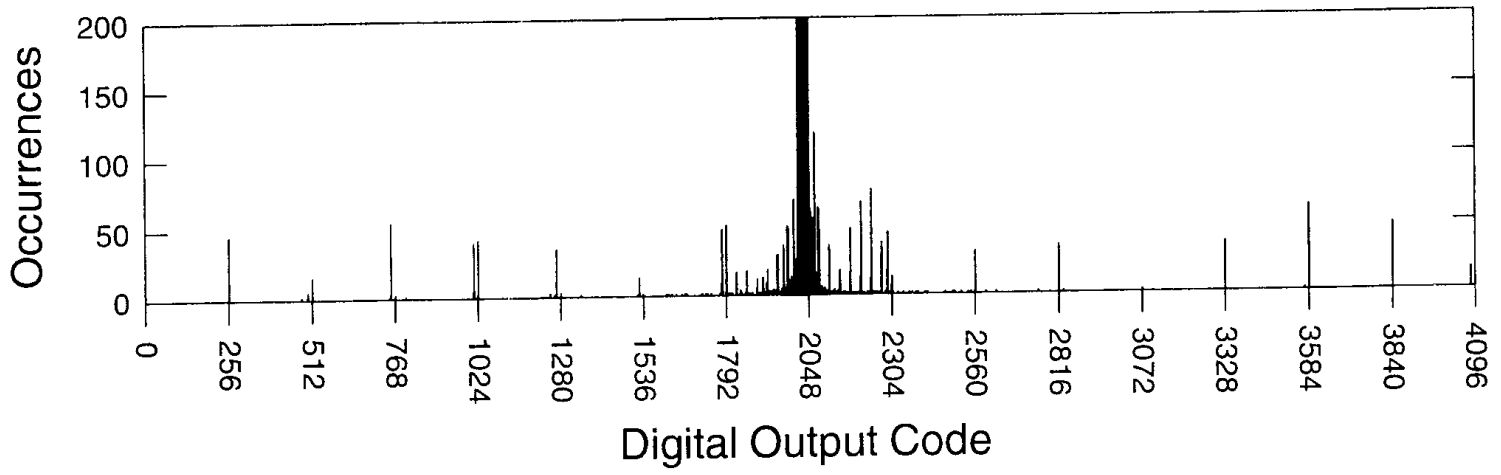

Figure 5. AD-42961 SN3302: $\mathrm{V}_{\mathrm{IN}}=0.0 \mathrm{~V}, \mathrm{~F}_{\mathrm{CLK}}=5 \mathrm{MSPS}$ Copper Ions, LET $=30 \mathrm{MeV}-\mathrm{cm}^{2} / \mathrm{mg}$

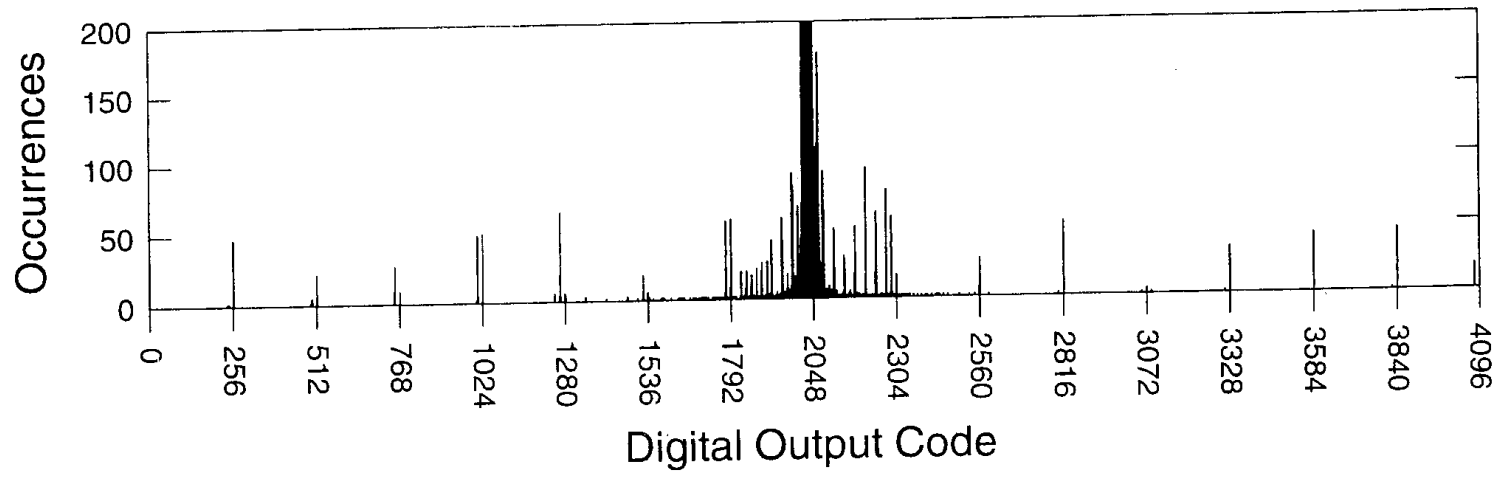

Figure 6. AD-42961 SN3302: $\mathrm{V}_{\mathrm{IN}}=0.0 \mathrm{~V}, \mathrm{~F}_{\mathrm{CLK}}=5 \mathrm{MSPS}$

Krypton Ions, $\mathrm{LET}=40 \mathrm{MeV}-\mathrm{cm}^{2} / \mathrm{mg}$

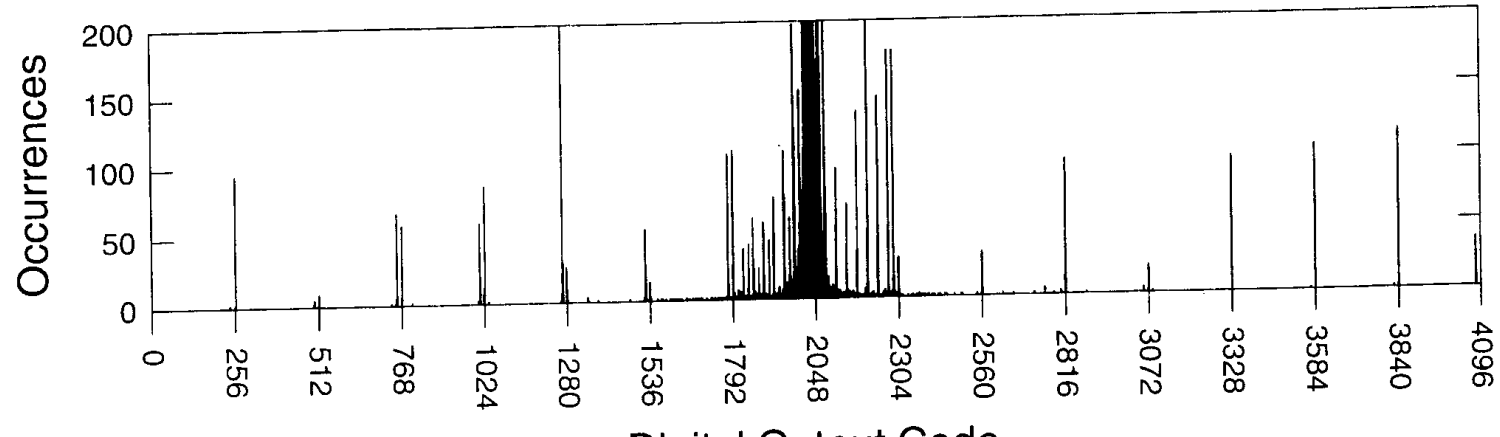

Digital Output Code

Figure 7. AD-42961 SN3302: $\mathrm{V}_{\mathrm{IN}}=0.0 \mathrm{~V}, \mathrm{~F}_{\mathrm{CLK}}=5 \mathrm{MSPS}$

Xenon Ions, $\mathrm{LET}=63.3 \mathrm{MeV}-\mathrm{cm}^{2} / \mathrm{mg}$ 
quantizers. The significance of these different errors and how they are analyzed are discussed in the next paragraph.

As was developed in the authors' 1990 paper [2], the raw data can be divided into two categories called "Noise" errors and "Offset" errors, which are analogous to the Gaussian and non-Gaussian errors discussed above. As will be shown, dividing the errors provides additional insights into the parts performance. For the AD-42961, the division between "Noise" and "Offset" errors was made at $+/-32$ bins from the expected data center bin, which is equivalent to $+/-16$ mVolts. In other words, all errors within $+/-32$ bins of the expected data are considered "Noise" errors, while errors with a magnitude greater than $+/-32$ bins are classified as "Offset" errors. The division may be unique for each part and is made both by analysis of the Gaussian distribution of the "Noise" errors and arbitrary selection of a valid division between signal and noise for system analysis purposes.

The second variation from static memory SEU test is to modify the cross section from static units of $\mathrm{cm}^{2} /$ device or $\mathrm{cm}^{2} /$ bit to a dynamic unit of $\mathrm{cm}^{2} /$ (million samples, MS). Because a high-speed ADC is only used dynamically, it is necessary to normalize the error cross section with respect to its unit output of a sample (or a million samples, MS, for convenience). To first order, this eliminates cross-section variation with $\mathrm{ADC}$ clock frequency.

Finally, two unique cross sections are calculated for each device, $\sigma_{\text {Noise }}$ for "Noise" errors and $\sigma_{\text {Offset }}$ for "Offset" errors. Both are calculated from equation 1, using the appropriate error category:

$$
\sigma=\frac{\text { number of errors }}{\text { fluence }\left(\# / \mathrm{cm}^{2}\right) \times \mathrm{F}_{\mathrm{clk}} \times \mathrm{t}_{\exp }}
$$

where $F_{c i k}$ is the ADC conversion rate in MSPS and $t_{\text {exp }}$ is the time, in seconds, the device is exposed to the ion beam.

The cross-section data for the 10 data sets described in Table 2 are shown in Table 3 . The ten rows of Table 3 are in the same sequence as Table 2 . Since that data is consistent within a factor of three, the average of the ten data sets, shown separately at the bottom, is plotted in Figure 8 . The error bars are contained within the datum markers of Figure 8 .

Figure 8 displays three cross-section curves to clearly illustrate the impact of error separation. The graph utilizes axes of $\log$ cross section versus linear LET as opposed to Petersen's new method of linear cross section versus log LET [5]. This is used to simplify graphical extraction of threshold and saturated cross section, but it is recognized that this datum does not strongly saturate. The "Raw" errors consist of all SEE-induced errors, with no separation. All cross-section data have units of $\mathrm{cm}^{2} / \mathrm{MS}$. Note that the "Raw" errors display no threshold and have a large "saturated" cross section. If only "Raw" error data were available for the device, it must be assumed that the probability of a maximum offset error (error data moving from the expected value to either 0 or 4095 , whichever has larger magnitude) is the same as that of the actual data being one or two bins from the expected data. The significance of these two errors to a system is clearly different. The line marked "Noise" errors, whose cross section is only marginally smaller than the "Raw" errors, contains only the errors that occurred within $+/-32$ bins of the expected data. If errors must exist, small magnitude errors will minimize system disruption. Again, no threshold is apparent. The third line is the "Offset" errors. "Offset" errors are distributed over the entire range of output codes in Figures 2-7, but the "Offset" curve of Figure 8 differs markedly from the "Raw" and "Noise" curves. First, note the shape of the curve displays a distinct threshold at about $10 \mathrm{MeV}-\mathrm{cm}^{2} / \mathrm{mg}$ (using $25 \%$ of the saturated cross section as the threshold), which does not exist in the "Noise" data. Second, even at high LET values, the magnitude of the cross section is at least a factor of five lower than the "Noise" data. This demonstrates that the probability of a large magnitude "Offset" error occurring is much smaller than that of a "Noise" error, a critical difference for system users.

The tight distribution of cross-section data across the various test variables, as shown in Table 3 , demonstrates that the experimental "Noise" and "Offset" cross sections of this device are independent of part bias, input voltage, and ion rate (flux). These are key results that validate the test and analysis techniques of references 1 and 2 . The "Noise" cross sections are also independent of clock frequency. The "Offset" error data for clock frequency variation (rows 8 and 9 in Table 3) are consistently higher than the other data, but still within a factor of three. Since it tracks the other data, it was included in the average data. Since this data tracks the body of data, it demonstrates that the same error mechanisms are present, but that some sub-set of the "Offset" errors are dependent upon ADC clock frequency. The limited data available precludes further analysis.

\section{PROTON TEST}

Limited proton data were recently collected on the AD-42961 at the Indiana University Cyclotron. Using $165 \mathrm{MeV}$ protons, data were collected at a fluence of $1.2 \mathrm{E} 11 \mathrm{ions} / \mathrm{cm}^{2}$. Applying the same techniques used for the heavy ion data, the "Noise" cross section, $\sigma_{\mathrm{N}}$, measured $1.4 \mathrm{E}-10 \mathrm{~cm}^{2} / \mathrm{MS}$, and the "Offset" cross section, $\sigma_{\mathrm{O}}$, measured 8.3E-14 $\mathrm{cm}^{2} / \mathrm{MS}$. Recall that a nuclear reaction creates higher LET fragments for one in every 1E5 to 1E6 protons. The "Offset" cross section demonstrates a continued fall-off in error rate from the nitrogen ion data of $3.7 \mathrm{E}-7 \mathrm{~cm}^{2} / \mathrm{MS}$; but note that "Offset" errors do still occur in protons. The "Noise" cross section is more interesting. The measured value of $1.4 \mathrm{E}-10 \mathrm{~cm}^{2} / \mathrm{MS}$ is just over five orders of 


\begin{tabular}{|c|c|c|c|c|c|c|c|c|c|c|c|c|c|c|c|c|c|}
\hline \multicolumn{4}{|c|}{ All Data $\mathrm{E}-5 \mathrm{~cm}^{2} / \mathrm{MS}$} & \multicolumn{3}{|c|}{$\mathrm{R}=\mathrm{Raw}$ Errors } & \multicolumn{4}{|c|}{$\mathrm{N}=$ Noise Errors } & \multicolumn{3}{|c|}{$\mathrm{O}=$ Offset Errors } & \multicolumn{4}{|c|}{$\ldots=$ No Data } \\
\hline \multicolumn{3}{|c|}{$\mathrm{N}$} & \multicolumn{3}{|c|}{$\mathrm{Ne}$} & \multicolumn{3}{|c|}{$\mathrm{Ar}$} & \multicolumn{3}{|c|}{$\mathrm{Cu}$} & \multicolumn{3}{|c|}{$\mathrm{Kr}$} & \multicolumn{3}{|c|}{$\mathrm{Xe}$} \\
\hline $\mathrm{R}$ & $\mathrm{N}$ & 0 & $\mathrm{R}$ & $\mathrm{N}$ & o & $R$ & $\mathrm{~N}$ & o & $R$ & $\mathrm{~N}$ & 0 & $\mathrm{R}$ & $N$ & o & $R$ & $\mathrm{~N}$ & o \\
\hline 1.9 & 1.9 & .04 & 2.7 & 2.5 & .22 & 4.1 & 3.6 & .53 & 5.8 & 4.9 & .91 & 7.4 & 6.3 & 1.1 & - & - & - \\
\hline 2.2 & 2.2 & .02 & 3.3 & 3.2 & .14 & 4.3 & 3.8 & .43 & 5.5 & 4.9 & .65 & 7.5 & 6.6 & .89 & 8.6 & 7.4 & 1.2 \\
\hline 2.7 & 2.6 & .03 & 3.3 & 3.1 & .19 & 4.9 & 4.3 & .56 & 5.9 & 4.9 & 1.0 & 7.8 & 6.7 & 1.1 & 9.4 & 7.5 & 1.9 \\
\hline 2.5 & 2.5 & .02 & 3.4 & 3.2 & .23 & 4.8 & 4.2 & .57 & 6.0 & 5.0 & .98 & 8.1 & 6.9 & 1.3 & 9.3 & 7.4 & 1.9 \\
\hline-- & - & - & 3.2 & 3.0 & .14 & 5.1 & 4.6 & .53 & 5.8 & 5.2 & .61 & 11. & 9.9 & 1.3 & 9.4 & 8.2 & 1.2 \\
\hline-- & - & - & 1.8 & 1.7 & .13 & 3.1 & 2.8 & .39 & 4.0 & 3.3 & .64 & 5.8 & 4.9 & .88 & 5.4 & 4.1 & 1.3 \\
\hline 1.7 & 1.7 & .03 & 2.2 & 2.1 & .17 & 3.5 & 3.1 & .42 & 4.5 & 3.8 & .70 & 6.8 & 5.9 & .91 & 7.6 & 6.4 & 1.2 \\
\hline 2.3 & 2.3 & .03 & 3.8 & 3.1 & .65 & 6.2 & 4.6 & 1.6 & 8.4 & 5.8 & 2.6 & 11. & 8.0 & 2.9 & 14. & 10. & 3.3 \\
\hline 1.6 & 1.6 & .03 & - & - & - & 4.0 & 3.3 & .70 & 5.9 & 4.8 & 1.2 & 7.6 & 6.3 & 1.3 & 11. & 9.2 & 1.7 \\
\hline 1.9 & 1.9 & .02 & 2.6 & 2.5 & .16 & 3.9 & 3.5 & .40 & 5.3 & 4.6 & .69 & 3.7 & 3.3 & .46 & 10. & 8.8 & 1.2 \\
\hline \multicolumn{18}{|c|}{ Average Data For Figure $8\left(\mathrm{~cm}^{2} / \mathrm{MS}\right)$} \\
\hline \multicolumn{3}{|c|}{$\mathrm{R}=2.14 \mathrm{E}-5$} & \multicolumn{3}{|c|}{$2.91 \mathrm{E}-5$} & \multicolumn{3}{|c|}{$4.73 \mathrm{E}-5$} & \multicolumn{3}{|c|}{$5.83 \mathrm{E}-5$} & \multicolumn{3}{|c|}{$7.88 \mathrm{E}-5$} & \multicolumn{3}{|c|}{$9.57 \mathrm{E}-5$} \\
\hline \multicolumn{3}{|c|}{$\mathrm{N}=2.11 \mathrm{E}-5$} & \multicolumn{3}{|c|}{$2.50 \mathrm{E}-5$} & \multicolumn{3}{|c|}{$4.12 \mathrm{E}-5$} & \multicolumn{3}{|c|}{$4.81 \mathrm{E}-5$} & \multicolumn{3}{|c|}{$6.65 \mathrm{E}-5$} & \multicolumn{3}{|c|}{$7.76 \mathrm{E}-5$} \\
\hline \multicolumn{3}{|c|}{$\mathrm{O}=3.71 \mathrm{E}-7$} & \multicolumn{3}{|c|}{$2.17 \mathrm{E}-6$} & \multicolumn{3}{|c|}{$5.95 E-6$} & \multicolumn{3}{|c|}{$1.02 \mathrm{E}-5$} & \multicolumn{3}{|c|}{$1.24 \mathrm{E}-5$} & \multicolumn{3}{|c|}{$1.71 \mathrm{E}-5$} \\
\hline
\end{tabular}

* See Table 2 for parametric information

\begin{tabular}{|c|c|c|c|}
\hline \multicolumn{4}{|c|}{$\begin{array}{c}\text { TABLE 4: SAMPLE ERROR RATE CALCULATIONS } \\
\text { (Error Rate }=\text { Error Count } x \text { conversions/day) }\end{array}$} \\
\hline Orbit & 300-km LEO & 4000-km Proton Belt & 35768-km Geosync \\
\hline "Noise" Error Count & 3.7E-2 Errors/MS-day & 1.2E-1 Errors/MS-day & 1.3E0 Errors/MS-day \\
\hline "Noise" Error Rate & 16,070 Errors/day & 49,680 Errors/day & 540,000 Errors/day \\
\hline "Offset" Error Count & 7.2E-6 Errors/MS-day & 3.0E-5 Errors/MS-day & 1.4E-4 Errors/MS-day \\
\hline "Offset" Error Rate & 3 Errors/day & 13 Errors/day & 62 Errors/day \\
\hline
\end{tabular}




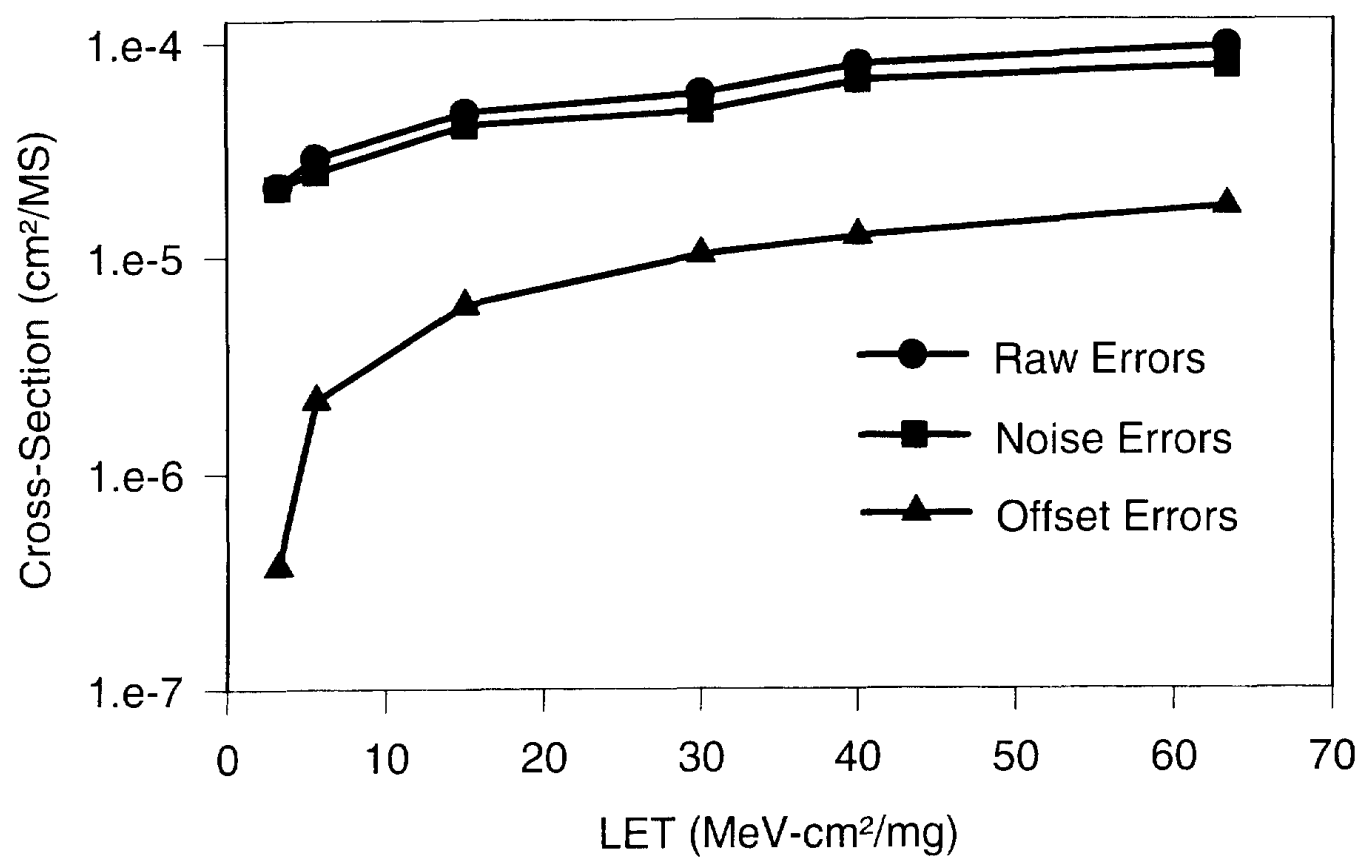

Figure 8. AD-42961 Average Cross Section vs. LET

magnitude less than the corresponding value found for nitrogen ions. This indicates that the rate of occurrence for "Noise" errors is the same for nitrogen ions and the secondary reactions of the protons. This demonstrates that "Noise" errors DO NOT exhibit an LET threshold within the LET spectrum of proton secondary nuclear reactions. The significance of this is discussed in the next section. Given that the proton "Noise" cross section is closer to $1 \mathrm{E}-5$ than $1 \mathrm{E}-6$ below the heavy ion cross section, an argument can be formulated (but certainly not proven) that a small number of direct proton upsets do indeed occur.

\section{SYSTEM IMPACT}

To demonstrate the potential system impact of SEE-induced errors in ADC, sample rate calculations for the heavy ion data have been made using "Space Radiation." The existing proton data are too limited to make a reasonable calculation. The three orbits of Table 4 were selected to demonstrate device performance in a variety of common orbits. All orbits use an inclination of 45 degrees, Adams $10 \%$ Worst Case Environment, and 50 mils of aluminum for all calculations. For the "Noise" error data, a threshold of $0.1 \mathrm{MeV}$ $\mathrm{cm}^{2} / \mathrm{mg}$ is assumed and a saturated cross section of $1 \mathrm{E}-4$ $\mathrm{cm}^{2} / \mathrm{MS}$ is extracted. The low threshold is assumed, but further validated by the proton test data. For the "Offset" error data, a threshold of $10 \mathrm{MeV}-\mathrm{cm}^{2} / \mathrm{mg}$ and a saturated cross section of $1.8 \mathrm{E}-5 \mathrm{~cm}^{2} / \mathrm{MS}$ are extracted, as was discussed.
Since the calculations were made using a dynamic cross section (with units of $\mathrm{cm}^{2} / \mathrm{MS}$ ), the direct computer output is an "error count" and not the traditional error rate associated with static RAM calculations. Device error rate is calculated by multiplying the error count by the number of conversions made by the ADC in a unit time (per day in this example). At the AD-42961's rated clock rate of 5 MSPS, running continuously, the device produces $4.32 \mathrm{E} 5$ million samples (MS) per day. Table 4 displays both the error count (in errors/(MS-day)), and the error rate (in errors/day) for three demonstration orbits.

Note the nearly four order of magnitude difference in "Offset" and "Noise" error counts. In any orbit, there are 4000 to 9000 "Noise" errors for each "Offset" error. Analog designers should note that the error repetition rate of the "Noise" errors is low, being less than six errors/second in the worst-case geosynchronous orbit. This demonstrates that the system will be required to deal with regular occurrences of both "Noise" and "Offset" errors in the ADC, but would be forced to overcompensate for large errors if error separation was not used. The impact of such errors is a strong function of the type of processing being done by the system. If all of the data is averaged, then individual SEEinduced errors will not strongly impact performance. If, on the other hand, data "sparkle" codes cause additional processor loading, the system impact of SEE errors may be severe. 


\section{ANALOG SEE}

The ADCs "Noise" and "Offset" cross-section curves shown in Figure 8 have fundamentally different characteristics. It is difficult to discuss both curves using a single model such as the ideal memory device model of a step function at a given threshold LET $\left(\mathrm{L}_{\mathrm{t}}\right)$. As has been discussed, the "Noise" cross section does not demonstrate a threshold LET. The great majority of these "Noise" errors are due to the direct collection of ion-induced charge by the sensitive analog circuitry of the ADC. Thus, the "Noise" errors are predominantly analog SEE and require a new model to explain their ideal response.

For memory cells, critical charge $\left(Q_{c}\right)$ is the accepted model. Total circuit response to ion-induced charge is modelled by both the circuit's properties and the charge deposited; but for a given model, one factor will be dominant. Critical charge is a behavioral model of circuit response and the circuit property $Q_{c}$ dominates. The deposited charge either exceeds $Q_{c}$ and the cell changes state or not. A step function is, thus, an adequate model of the ideal response of a memory cell. By extension, the ideal model of the memory chip response is also a step function at a given $L_{\mathrm{r}}$. Circuit $\mathrm{Q}_{\mathrm{c}}$ appears to be an adequate model for "Offset" errors in an ADC.

To model analog circuit response, the quantity of charge deposited at a node is the dominant variable; and a device level model defines the amplitude and duration of the transient pulse generated at the circuit node. The circuit impedance acts to scale the resultant node voltage. The extension of the SEE-induced transient signal to the macrocell or circuit level is more difficult for analog and mixedsignal circuits. The transient may be codified by the downstream circuitry as a legitimate (if undesired) signal, or it may not propagate and become unobservable. In the case of the AD-42961 ADC, the analog SEEs are codified by the quantizers as "Noise" errors. Total circuit response will be architecture-dependent and does not extend simply from the node response.

Analog SEE must be modelled at the device level. The model is dominated by the quantity of charge deposited and moderated by the circuit impedance. Critical charge and threshold LET are not defined. A corollary is that the ideal response of analog SEE is not a step function and is circuitdependent with the linear relationship between charge and node capacitance often a major factor. This model explains the characteristics of the "Noise" cross-section curve of Figure 8 .

\section{CONCLUSIONS}

Several significant results have been demonstrated. The test and analysis technique used produces a dynamic cross section independent of part bias, input voltage, and ion rate (flux). The independence of dynamic cross section and clock frequency is partially demonstrated. The "Offset" cross sections are lower clock frequencies are consistently slightly higher than the other data. This will require further data to analyze. The separation of SEE errors into "Noise" and "Offset" errors greatly enhanced the interpretation of the test data. The devices did not latch under any tested condition. Sample error rate calculations demonstrate that SEE-induced errors will be a regular occurrence in many orbits, and the effect of the errors on system performance must be analyzed and anticipated during system design.

Finally, analog SEE is shown to require a more complex device level model to replace the simple behavioral model used for memory cells. Analog SEE is a result of direct charge collection and is not dependent upon a circuit $Q_{c}$.

\section{ACKNOWLEDGEMENTS}

The authors wish to thank Virgil Strahan for test assistance and discussions on system impact. Dr. Edward Petersen provided review and thoughtful insights on many occasions.

\section{REFERENCES}

1. T. L. Turflinger and M. V. Davey, IEEE Trans. Nucl. Sci., Vol NS-36, p2356, (1989).

2. T. L. Turflinger and M. V. Davey, IEEE Trans. Nucl. Sci., Vol NS-37, p1832, (1990).

3. Analog Devices, Inc, 1994 Military/Aerospace Reference Manual, p6-51, p6-55.

4. MADCAT Tester, developed by Mission Research Corporation under contract to Crane Division, NSWC.

5. E. L. Petersen, J. C. Pickel, J. H. Adams, Jr., and E. C. Smith, IEEE Trans Nucl. Sci., Vol NS-39, p1577, (1992). 